\title{
CONFORMAL PHASED ARRAY WITH BEAM FORMING FOR AIRBORNE SATELLITE COMMUNICATION
}

\author{
H. Schippers (schipiw@nlr.nl), J. Verpoorte , P. Jorna, A. Hulzinga \\ (National Aerospace Laboratory NLR, Amsterdam), \\ A. Meijerink, C. Roeloffzen, (University of Twente, Enschede), \\ R.G.Heideman, A. Leinse, (LioniX bv, Enschede), \\ M. Wintels (Cyner Substrates, Utrecht)
}

\begin{abstract}
For enhanced communication on board of aircraft novel antenna systems with broadband satellite-based capabilities are required. The installation of such systems on board of aircraft requires the development of a very low-profile aircraft antenna, which can point to satellites anywhere in the upper hemisphere. To this end, phased array antennas which are conformal to the aircraft fuselage are attractive. In this paper two key aspects of conformal phased array antenna arrays are addressed: the development of a broadband Ku-band antenna and the beam synthesis for conformal array antennas. The antenna elements of the conformal array are stacked patch antennas with dual linear polarization which have sufficient bandwidth. For beam forming synthesis a method based on a truncated Singular Value Decomposition is proposed.
\end{abstract}

\section{INTRODUCTION}

For enhanced communication on board of aircraft novel antenna systems with broadband satellite-based capabilities are required. The technology must bring live weather reports to pilots, as well as live TV and high-speed Internet connectivity to passengers. Satellite communication services can be provided by Low Earth Orbiting (LEO) systems and Geostationary (GEO) systems. Today there are more than 300 operational geostationary satellites. These satellites are being used for television broadcasting, communications and weather forecasting. In general, receive and transmit antennas of ground stations do not need to track geostationary satellites. These antennas are installed at a fixed location and are much less expensive than tracking antennas. However, for a mobile terminal for satellite communication (for instance when applied on an aircraft) a tracking antenna is required in all circumstances. Many studies are going on worldwide to employ these Ku-band geostationary satellites for communication with mobile terminals on cars, trains, ships and aircraft.

$\mathrm{Ku}$-band terminals on board of moving platforms require antennas with high gain of which the main beam can continuously be steered to geostationary satellites. To this end the following types of antenna systems can be identified:

- Mechanically steered reflector antennas (see Figure 1).

- Mechanically steered array antennas (beam of array is fixed, but mechanically steered).
- Electronically steered phased array antennas (see Figure 7).

- Hybrid steered antennas; such antennas are normally mechanically steered in one direction (e.g. azimuth), and electronically in the other direction (e.g. elevation).

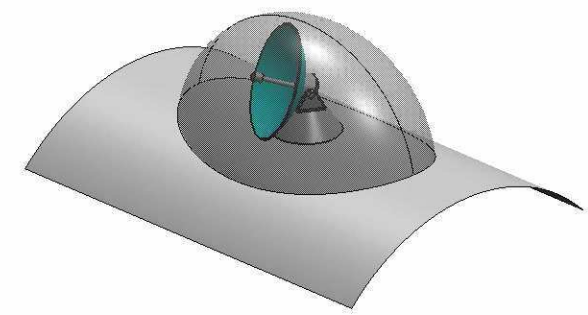

Figure 1 Mechanically steered reflector antenna

Mechanically steered reflector and array antennas require a large radome for reasons of protection and aerodynamic behaviour. Such a radome increases the aerodynamic drag and requires additional cuts and stiffeners in the fuselage structure. Therefore, since many years research is being performed to develop electronically steered phased array antennas (or hybrid antennas) on singly curved structures, for instance conformal to the fuselage of an aircraft. These conformal antennas should have similar antenna characteristics as mechanically steered antennas, with high scan capabilities at high latitudes. In addition the antenna should be able to withstand severe environmental conditions such as temperature, pressure and vibration.

In the Netherlands, a consortium (consisting of the University of Twente, Lionix BV, the National Aerospace Laboratory NLR and Cyner Substrates) is developing technology for such a conformal airborne antenna. The hardware for this antenna consists of a broadband conformal Ku-band phased array antenna (developed by NLR and Cyner) and a broadband optical beam forming network with True Time Delays (developed by the University of Twente and Lionix). Beam forming synthesis methods are being developed by NLR to control the beam shape and direction. This technology is developed in the national project FlySmart. The objective of this paper is to present research results of two key topics: the broadband conformal phased array antenna and the array antenna beam forming synthesis method. 
The antenna front-end consists of a broadband conformal phased array antenna (bandwidth $\geq 2 \mathrm{GHz}$ ) operating in Ku-band. The antenna elements are stacked patch antennas with dual linear polarization. First results indicate sufficient bandwidth in input impedance and radiation pattern. Special attention is paid to the manufacturability of the antenna by selecting specific materials and appropriate fabrication techniques.

Once the global design of the conformal phased array has been established, further research concerns the verification of its beam forming capabilities. For instance, is it possible to find an optimum set of element excitations for the antenna elements of the array to best satisfy the radiation pattern demands in some well-defined sense and to steer the beam in the direction of the geostationary satellites? The National Aerospace Laboratory NLR has developed an adapted least squares pattern synthesis that yields an efficient tapering for conformal phased arrays (see [1]). For the steering of the beam of the conformal phased array a squint-free, continuously tunable mechanism is proposed that is based on a fully integrated optical beam forming network (OBFN) using cascades of optical ring resonators (ORRs) as tunable delay elements [2].

\section{SYSTEM ASPECTS}

In the ITU Radio Regulations [3] portions of the Ku-band are allocated to aeronautical services:

- $\quad$ AES receive band 1: $10.70-11.70 \mathrm{GHz}$ (primary allocation to fixed satellite service)

- $\quad$ AES receive band 2: $12.50-12.75 \mathrm{GHz}$ (primary allocation to fixed satellite service)

- AES transmit band: $14.00-14.50 \mathrm{GHz}$ (secondary allocation to mobile satellite service)

The Aeronautical Earth Stations (AES) have to comply with ITU-R RECOMMENDATION M.1643 [4] and with ETSI EN 302186 [5], a harmonised European Norm for satellite mobile Aircraft Earth Stations (AESs) operating in the $11 / 12 / 14 \mathrm{GHz}$ frequency band.

In addition, reception of commercial satellite broadcasts is of interest:

- Satellite TV: $11.70-12.50 \mathrm{GHz}$ (primary allocation to broadcast satellite service)

In the Dutch FlySmart project, only the receive antenna system will be developed. The objective is to develop a conformal phased array antenna having an instantaneous bandwidth of $2 \mathrm{GHz}$, covering the whole frequency range of 10.7 to $12.75 \mathrm{GHz}$.

Satellites operating in these bands are geostationary satellites spaced $2^{\circ}$ apart in the United States and $3^{\circ}$ in Europe. In order to be able to receive these satellites also at high latitudes (e.g. during inter-continental flights) the antenna system should have sufficient performance at low elevation angles.

Therefore the antenna system is required to have a small beamwidth (to discriminate between the satellite signals) and a high gain ( $>30 \mathrm{~dB})$ also at low elevation angles. Since gain of the antenna is related to the effective aperture of the antenna in the direction of the satellites, a conformal antenna also covering side parts of the fuselage could be an advantage.
The phased array antenna shall maintain the proper (linear) polarization during all attitudes and at all positions of the aircraft (also at high latitudes).

An antenna to be used on aircraft has to be able to operate in severe environmental conditions concerning temperature, pressure, vibration and humidity. The environmental requirements for civil airborne equipment are given in RTCA DO-160 or EUROCAE ED-14 [6].

In general the antenna system consists of a phased array antenna, electrical-to-optical conversion, optical beam forming (or beam steering) and optical-to-electrical conversion (Figure 2)

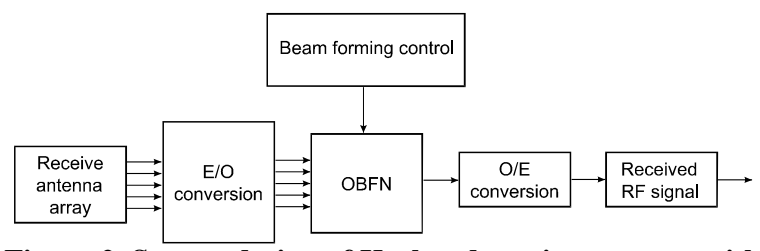

Figure 2 System design of $\mathrm{Ku}$-band receive antenna with Optical Beam Forming Network (OBFN)

The phased array antenna will be a conformal (singly-curved) 2-D array of dual linear polarised broadband antenna elements. Each antenna is followed by a Low Noise Amplifier (LNA) and down-converter (together a Low Noise Block converter, LNB). The Local Oscillator (LO) signals of the LNBs are synchronised to maintain an appropriate phase relation between the OBFN channels. The Intermediate Frequency (IF) signal from the LNB is subsequently fed to optical modulators which perform the electrical-to-optical conversion. In the Optical Beam Forming Network (OBFN) each individual signal is attenuated and delayed in order to shape and direct the antenna beam. The sum of all signals is converted back from the optical to the electrical domain.

The tracking algorithm will use the aircrafts position and attitude to determine the appropriate polarization and azimuth and elevation of the antenna beam. To reach the objective of a $2 \mathrm{GHz}$ bandwidth, both the antenna front-end and the beam forming network should have broadband characteristics. Therefore, the antenna front-end consists of an array of stacked patch antennas. The beam forming network consists of an optical network with True Time Delays (TTD) which have inherently a large bandwidth. To have a $2^{\circ}$ beamwidth and high gain antenna (approx. $36 \mathrm{~dB}$ ), a large array antenna is needed. The array size may be in the order of 40 by 40 antenna elements. 


\section{DEVELOPMENT OF KU-BAND ANTENNA}

The objective is to develop a very low-profile aircraft antenna, which can point to geostationary satellites anywhere in the upper hemisphere. Traditionally, reflectorbased solutions have been proposed which are unattractive to airlines since they create significant drag and push up fuel costs. Instead conformal phased array antennas of which the main beam can electronically be steered are recommended. In general an array antenna consists of a multiple of active antenna elements coupled to a common source to produce a directive radiation pattern. The antenna element could be any type. Since the aircraft antenna should have a low profile most suitable antenna elements are microstrip patches, which are fed by apertures in a ground plane. The main disadvantage of these microstrip patch antennas is their limited bandwidth which is in the order of a few percent for a typical patch radiator. This property makes the classical patch antennas less attractive for broadband satellite communication. In order to increase the bandwidth, stacked patches have been advocated in literature. In the stacked patch configuration a parasitic element is placed above a lower patch, separated by foam or other space filler. In this manner, bandwidths on the order of $30-35 \%$ can be achieved [7]. In this section we present the design of stacked patch $\mathrm{Ku}$-band antenna element and we discuss the preliminary design of a conformal array of stacked Ku-band antenna elements.

\subsection{Design of single Ku-band antenna element}

A common approach for increasing the bandwidth is to add parasitic elements to the antenna structure, e.g. a stacked patch. This reduces the impedance variation of the antenna with the frequency, thus enhancing bandwidth performance. Various arrangements of stacked structures have been investigated in [8] and [9]. In practice, it is difficult to optimise the bandwidth of these structures due to their sensitivity with respect to many physical parameters (patch sizes, substrate thicknesses, and feed-point position). Research has focused on the choice of the materials for the dielectric layers in the stacked configuration. Thick laminates of low permittivity provide the largest bandwidth and surface wave efficiency (see [9]).

Figure 3 shows the design of the present Ku-band antenna element, consisting of a multilayer structure where the parasitic and radiating patches are mounted on commercial available Duroid substrates. The space between the patches is filled with typical space filler that is being developed for this purpose. The lowest patch is being fed by an aperture in a lower ground plane, again mounted on a substrate. On the lower side of this substrate are horizontal feed lines, which are connected to shielded vertical feed lines to provide connections with the beam forming network on a lower layer.

The dimensions of the Ku-band antenna design have been optimised by using ANSOFT HFSS simulation software [10]. The ANSOFT HFSS model for the design is shown in Figure 4. The dimensions of the patches, dog bone aperture and thicknesses of foam layers have been optimised with the aim to get an antenna which could span the $\mathrm{Ku}$ band from 10.7 to $12.75 \mathrm{GHz}$. Some results of this optimization process are shown in Figure 5 and Figure 6.
Figure 5 shows the computed radiation pattern for left-hand and right-hand circular polarization for two sections in the hemisphere (at $\varphi=0$ and $\varphi=90$ degrees). The circular polarization is obtained by feeding both linear inputs $90^{\circ}$ out of phase. The computed gain of this stacked patch antenna is about $9 \mathrm{dBi}$. Figure 6 displays the reflection coefficients of both inputs of the antenna element. Notice that the reflection is below $-10 \mathrm{~dB}$ in the frequency range between 10.7 to $12.75 \mathrm{GHz}$ as aimed, which indicates that this element has sufficiently large bandwidth for broadband data transmission.

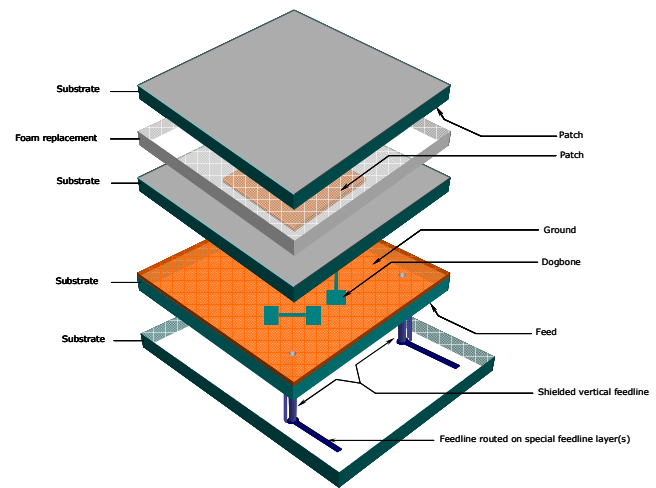

Figure 3 Design of stacked Ku-band antenna element

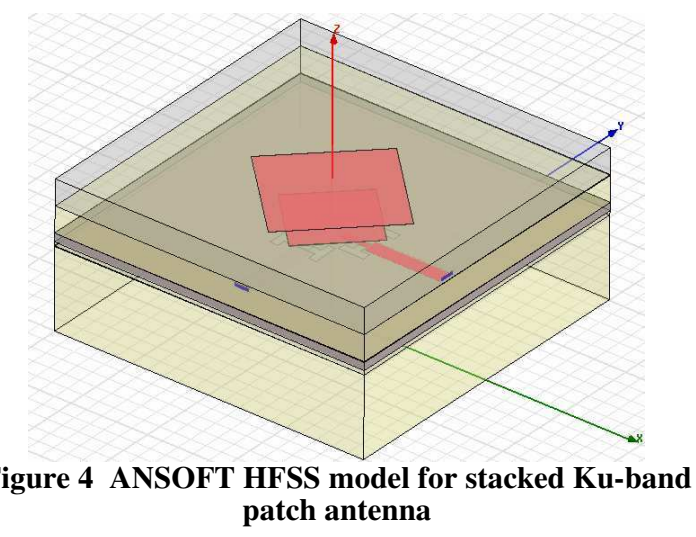

3.2. Prelimary design of Ku-band band antenna array The Ku-band antenna array will consist of a rectangular array of antenna elements (as presented in the previous section) mounted on a circular cylindrical aluminium surface (hence singly curved). The distance between the antenna-elements will be approximately $11.8 \mathrm{~mm}$ (measured between the centres of the antenna-elements).

The antenna has to be curved equal to the fuselage of an aircraft. Hence, the outer convex surface is placed under substantial tension and is forced to stretch. This stretching causes a non-flexible (foam with resin absorption) material to crack. Furthermore, the bending can result in deformation and tearing of the copper antenna elements, which will pull the antenna apart. Therefore, the antenna will be faceted in the curved direction. Each face will support a separate rectangular array of $\mathrm{Ku}$-band antenna 
elements (with only a few elements in azimuth direction). On the upper side of the antenna structure small notches will be made to provide the curvature. Preliminary vibration tests using a sample structure were promising.

Two planar samples with Ku-band arrays have been manufactured. The first sample contains an array of $8 \times 1$ antenna-elements, the second sample had $8 \times 8$ elements.

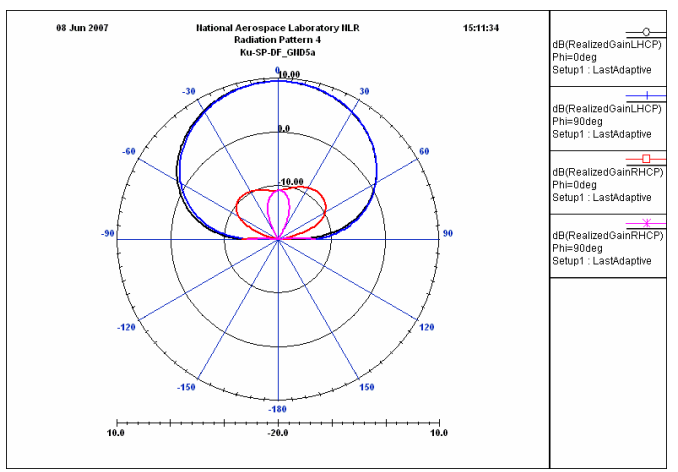

Figure 5 Radiation patterns for stacked Ku-band patch antenna (linear co-and cross polar components)

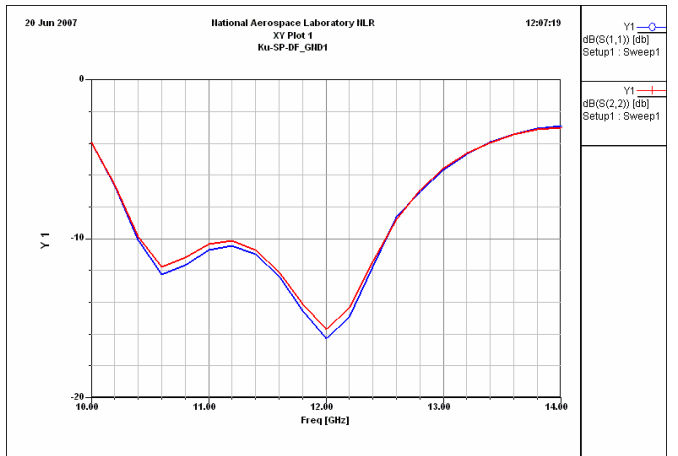

Figure 6 Reflection coefficients of stacked Ku-band patch antenna

\section{BEAM SYNTHESIS FOR CONFORMAL ARRAY ANTENNAS WITH EFFICIENT TAPERING}

\subsection{Introduction}

Phased array antennas contain many design parameters like the shape of the array, dimensions, number of elements, lattice, and type of radiating elements. Once the global design of the phased array has been established, further research concerns the verification of its beam forming capabilities. For instance, is it possible to find an optimum set of element excitations for the antenna elements of the array to best satisfy the radiation pattern demands in some well-defined sense? To this end, so-called beam synthesis techniques are developed. A survey of well-known synthesis techniques for conformal arrays is given in [11].

In this paper a beam synthesis technique for conformal phased array antennas with efficient tapering is presented. The excitation values of the array elements can be determined by solving an over-determined system of equations that minimizes the difference between a desired radiation pattern and a synthesized pattern in some sense. The classical least squares solution yields alternating excitation values resulting in low antenna taper efficiency. In this paper, it will be shown that a relatively simple adjustment to the classical least squares method will improve the taper efficiency. Here, the over-determined system of equations is solved by computing the pseudoinverse of a truncated Singular Value Decomposition. Synthesis techniques of practical importance need a mechanism which precludes unfeasible solutions. Most elegant are perhaps those techniques which impose constraints on the solution vector. In [12], [13] and [14] Vaskelainen describes phase synthesis by invoking constrained least-squares techniques with predefined amplitudes; these techniques, however, do not leave any room for amplitude optimization. In [15] and [16], convex optimization is applied, and it is demonstrated that this can easily incorporate equality constraints, level constraints on the amplitudes, quadratic constraints such as power constraints and upper bound constraints on the radiation pattern. Therefore, convex optimization algorithms are suitable for antenna pattern synthesis, at the expense, however, of sizeable computational effort. Since phase synthesis and power pattern synthesis yield non-convex problems, they require other optimization techniques like those described by Vaskelainen in [12], [13] and [14]. A power pattern synthesis approach based on semi-definite programming is presented in [17] and [18].

The starting point for the beam synthesis is to define an over-determined system of equations where the synthesized far-field pattern of the array agrees with a desired radiation pattern for a range of sample directions. The excitation values of the array elements can be determined by using a classical least squares method, where the least squares difference between the desired and the synthesized pattern is minimised. This method, however, may yield alternating excitation values resulting in an antenna with low taper efficiency. The taper efficiency indicates how efficiently the physical area of the antenna is utilized and how much directivity can be expected from the array taper compared to a broadside planar array. The objective of this paper is to present a slightly adapted least squares pattern synthesis that yields an efficient tapering for conformal phased arrays. The over-determined system of equations is solved by computing the pseudo-inverse of a truncated Singular Value Decomposition (SVD). In this paper, the method is applied to synthesize the beams of a circularly shaped phased array in Ku-band on a singly curved structure. However, it is foreseen that the pattern synthesis using truncated SVD of pseudo-inverses has a much wider range of applicability. 


\subsection{Least-Squares Method and Pseudo-Inverse}

The far-field radiation pattern of a general antenna array in the direction of the unit vector û can be written in the form:

$$
E(\hat{u})=\sum_{n=1}^{N} a_{n} g_{n}(\hat{u}) e^{j k_{0} \vec{r}_{n} \cdot \hat{u}}
$$

where $\vec{r}_{n}$ is the position vector and $a_{n}$ the complex excitation amplitude of the $\mathrm{n}^{\text {th }}$ element, $k_{0}$ is the wave number $2 \pi / \lambda_{0}$ with $\lambda_{0}$ the free space wavelength and $g_{n}(\hat{u})$ is the directivity of the $\mathrm{n}^{\text {th }}$ element in the direction of the unit vector $\hat{u}$. If $\hat{u}_{k}$ represents the direction $\left(\theta_{k}, \varphi_{k}\right)$ in polar coordinates and $x_{n}$, $y_{n}$ and $z_{n}$ are the coordinates of the position vectors $\vec{r}_{n}$, one can write (1) in $K$ sample directions in matrix form:

$$
\vec{E}=X \vec{A}
$$

Where

$$
\begin{aligned}
\vec{E} & =\left[E\left(\theta_{1}, \varphi_{1}\right) \cdots E\left(\theta_{K}, \varphi_{K}\right)\right]^{T} \\
\vec{A} & =\left[a_{1} \cdots a_{N}\right]^{T}
\end{aligned}
$$

are the far-zone electric field vector, and the excitation vector of the array, respectively. The elements $X_{k n}$ of the geometry-dependent matrix $X$ are given by:

$$
X_{k n}=g_{n}\left(\theta_{k}, \varphi_{k}\right) e^{j k_{0}\left(\sin \left(\theta_{k}\right) \cos \left(\varphi_{k}\right) x_{n}+\sin \left(\theta_{k}\right) \sin \left(\varphi_{k}\right) y_{n}+\cos \left(\theta_{k}\right) z_{n}\right)}(4)
$$

The radiated far field $\vec{E}$ has to match a desired electric field $\vec{D}$ by finding appropriate values for the excitation values of vector $\vec{A}$. Using equation (2) we obtain the following overdetermined set of $\mathrm{K}$ linear equations with $\mathrm{N}$ unknowns:

$$
X \vec{A}=\vec{D}
$$

The classical least-squares solution of (5) reads

$$
\vec{A}=\left(X^{H} X\right)^{-1}\left(X^{H} \vec{D}\right)
$$

where the superscript $\mathrm{H}$ denotes the Hermitian transpose. An expression for the least-squares solution can also be obtained by multiplying the right-hand side of (5) with the so-called pseudo-inverse. Let the SVD of matrix $X$ be given by

$$
X=U \Sigma V^{H}
$$

In (7) $U$ is a $K$ by $K$ unitary matrix which contains the leftsingular eigenvectors, $V$ is a $N$ by $N$ unitary matrix which contains the right-singular eigenvectors, and $\Sigma$ is the diagonal matrix containing the singular values of the lefthand side matrix, arranged in descending order:

$$
\sigma_{1} \geq \sigma_{2} \geq \ldots \geq \sigma_{N} \geq 0
$$

The pseudo-inverse of the left-hand side matrix in (5) is the $N$ by $K$ matrix:

$$
X^{+}=V \Sigma^{+} U^{H}
$$

with $\Sigma^{+}$given by

$$
\Sigma^{+}=\left[\operatorname{diag}\left(1 / \sigma_{1}, \cdots, 1 / \sigma_{N}\right) \quad 0\right]
$$

The least-squares solution using the pseudo-inverse of the SVD may be written as:

$$
\vec{A}=V \Sigma^{+} U^{H} \vec{D}
$$

On one hand, expression (11) shows that small singular values may cause small changes in the right-hand side of (5) to result in dramatic changes in the least-squares solution. On the other hand, large adjustments in the amplitude profile may only contribute marginally to a better radiation pattern. In [19] it is explained in detail how the sensitivity of the least-squares solution depends on the condition number of the rectangular matrix $\mathrm{X}$ in the left-hand side of (5). Despite our assumption that the rank of the rectangular matrix is N, its columns may be nearly dependent, and hence the singular values can be very small. To get rid of the huge amplitudes that do not contribute to a significantly better synthesized radiation pattern, it is proposed to compute the pseudo-inverse based on the truncated SVD, that is, to discard all singular values below a certain threshold $\sigma_{\min }$. Discarding too many singular values will result in a deteriorated radiation pattern, so the cut off threshold must be chosen with care. There are different strategies to find an optimal threshold. Once the full SVD is determined, the computational effort to evaluate the influence of the different cut-off values on the excitation coefficients and the radiation pattern is limited.

\subsection{Synthesis Example}

In this example we study the synthesis for a phased array operating in the 10.7-12.75 GHz Ku-band. With $\lambda_{\max }$ we denote the largest wavelength corresponding to a frequency of $10.7 \mathrm{GHz}$ and with $\lambda_{\text {mid }}$ we denote the wavelength corresponding to a frequency of $11.7 \mathrm{GHz}$. First a very simple straightforward synthesis for an array pattern of a large circularly shaped planar array of radius $10 \lambda_{\max }$ with a square lattice and an inter-element distance of $0.55 \lambda_{\text {mid }}$ is performed by conventional sampling of the continuous oneparameter circular aperture distribution described by Hansen in [20]. The parameter in this distribution is set to obtain a side-lobe ratio of $30 \mathrm{~dB}$. As element radiators we have taken circularly polarized circular patch antennas. The radiation 
patterns of these elements are approximated by the lowest order circular patch model as described in [21].

Next, the circularly shaped array is integrated into the fuselage of a Fokker 100. This is modelled by folding the circularly shaped array over the top of a cylinder of radius $1.65 \mathrm{~m}$ as is illustrated in Figure 7.

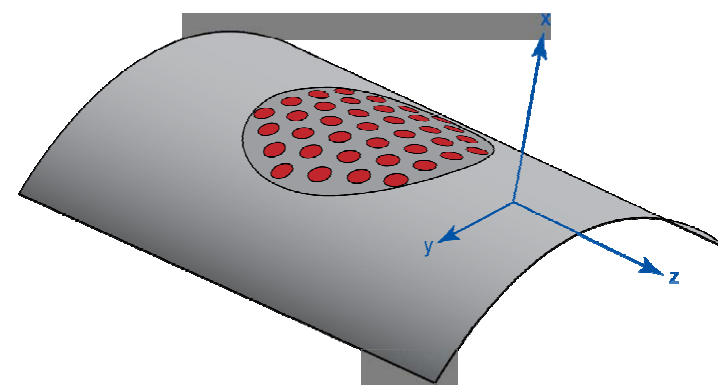

Figure 7 Model of a circularly shaped phased array antenna integrated in the fuselage of an aircraft

Now let us determine the excitation coefficients of the modestly curved 1237 element array with the least-squares method where the radiation pattern of the original planar disc array is taken as the desired radiation pattern. The synthesis will be performed with 16002 sample directions uniformly distributed over the radiation sphere. Since it is not our primary goal to do an in-depth analysis on the frequency and polarization optimization, we have chosen to reduce the computational burden and use only one polarization direction and only one frequency in the synthesis

Next, we investigate the effects of discarding the singular values below a chosen treshold $\sigma_{\min }$. More precisely, we will investigate changes in the element amplitude profile and in the corresponding radiation pattern. A very useful tool in evaluating the synthesis results is the taper efficiency. For curved arrays, the standard definition of taper efficiency used for linear and planar arrays needs to be modified, as is explained by Elliot in [22]. We have adopted his approach and use the following formula for the taper efficiency:

$$
\mathcal{E}_{T}=\frac{1}{N} \frac{\left|\sum_{n=1}^{N} a_{n} g_{\mathrm{co}, n}\left(\hat{u}_{\mathrm{scan}}\right) e^{j k_{0} \vec{r}_{n} \cdot \hat{u}_{\mathrm{scan}}}\right|^{2}}{\left(g_{\text {max }}\right)^{2} \sum_{n=1}^{N}\left|a_{n}\right|^{2}}
$$

In this formula $\hat{u}_{\text {scan }}$ is the unit vector in the direction of the main beam, $g_{c o, n}$ is the co-polarization component of the $n^{\text {th }}$ element and $g_{\max }$ is the maximum magnitude of the element field pattern.

In Figure 8 azimuthal cuts of the prescribed planar array pattern and the synthesized patterns as obtained with the least-squares solution and the least-squares approximate solution are shown. Obviously, both synthesized patterns cannot satisfy the desired pattern at angles far away from broadside, but this is unavoidable. Interestingly enough, the difference in the synthesized patterns is hardly distinguishable, but there is a major improvement in the taper efficiency for the approximate least-squares solution using a truncated SVD. The taper efficiency corresponding to the least-squares solution is $-15.7 \mathrm{~dB}$, unacceptably low, whereas the taper efficiency corresponding to the approximate least-squares solution is $-1.22 \mathrm{~dB}$ which is very good given the $-1.19 \mathrm{~dB}$ taper efficiency of the original excitation of the planar array. The low taper efficiency corresponding to the least-squares solution is caused by the large ratio of the largest to the smallest singular value. For this particular example, the largest singular value is $\mathbf{5 7 . 2}$ whereas the smallest singular value is 0.00456 . The approximate least-squares solution was computed by cutting of all singular values below a tenth of the maximum singular value. It can be demonstrated that discarding more singular values will not improve the taper efficiency significantly. Another indication that the amplitude profile has improved considerably is the maximum amplitude which is a factor 7.6 lower compared to the classical least-squares solution.

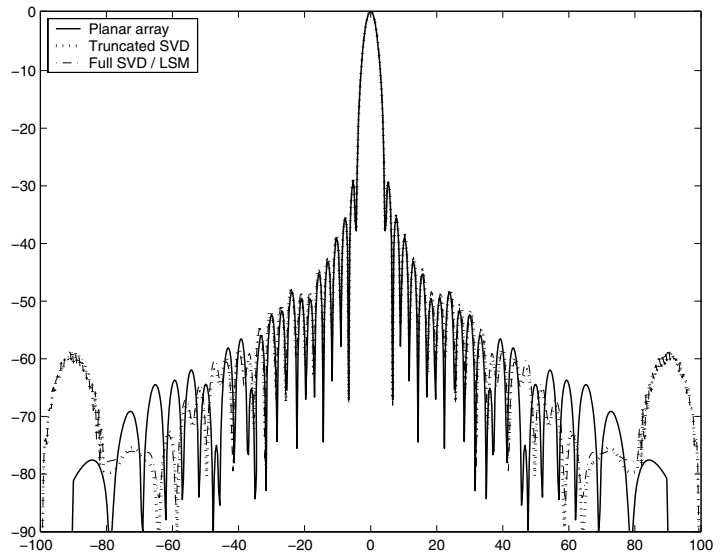

Figure 8 Destination pattern and synthesized patterns obtained with the least-squares solution and the leastsquares approximate solution for a broadside scan

Figure 9 shows azimuthal cuts of a prescribed planar array pattern, and the synthesized patterns obtained with both the least-squares solution and the least-squares approximate solution. Here, the desired pattern has a $40^{\circ}$ scan angle away from broadside. Again both synthesized patterns cannot satisfy the desired pattern at angles near $90^{\circ}$, as expected. The difference in the synthesized patterns is hardly distinguishable, but the taper efficiency has again improved substantially for the approximate least-squares solution. The taper efficiency corresponding to the least-squares solution is unacceptably low, $-19.1 \mathrm{~dB}$, whereas the taper efficiency corresponding to the approximate least-squares solution is $-5.52 \mathrm{~dB}$ which is very good in comparison to the $-5.41 \mathrm{~dB}$ taper efficiency of the original excitation of the planar array. 


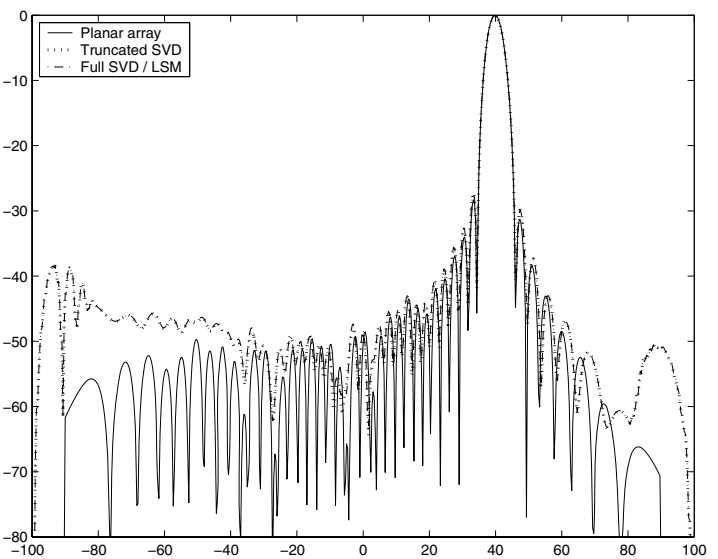

Figure 9 Destination pattern and synthesized patterns obtained with the least-squares solution and the leastsquares approximate solution for a scan $40^{\circ}$ away from broadside

\subsection{Conclusions and remarks on the beam forming synthesis}

The classical least squares pattern synthesis method may yield unfeasible excitations for the antenna elements of phased arrays. The taper efficiency may be very low. This is likely related to the large condition number occurring in the classical SVD, i.e. the ratio between the maximum and minimum singular values may be large. A relatively simple adaptation of the classical least squares synthesis yields a solution with an improved amplitude profile. Truncate the SVD (i.e. discard the smallest singular values) and the corresponding pseudo-inverse yields excitation values with much better taper efficiency while the radiated pattern closely resembles the classical least squares synthesised pattern obtained with the pseudo-inverse of the full nontruncated SVD.

It should be noted that for this particular example, with a desired pencil beam pattern derived from a planar disc array and a conformal array that is obtained by introducing a very modest curvature into the original array, a simple projection synthesis method with proper adjustment of the excitation phases may yield very good results as well but it is clearly demonstrated that applying the least-squares method blindly can give unsatisfactory results. For more complicated desired patterns the simple projection method may yield poor results. Further research is recommended to investigate the applicability of the truncated SVD approach for the synthesis of more complicated patterns of conformal arrays integrated on singly and doubly curved structures.

\section{CONCLUSIONS}

For broadband satellite communication on board of aircraft the development of an advanced conformal antenna array has been discussed. Such antennas require high gain, small beamwidth and large bandwidth. The beam shall be steered continuously in the direction of the communication satellite. To reach the objective of a $2 \mathrm{GHz}$ bandwidth at $\mathrm{Ku}$-band, both the antenna front end and the beam forming network should have broadband characteristics.

Ku-band antenna elements with sufficiently large bandwidth have been presented. The Ku-band antenna element is a stacked patch configuration where a parasitic element is placed above a lower patch, separated by dedicated space filler. The first manufactured prototype antennas indicate that the bandwidth is sufficiently large. Furthermore, the required gain can be achieved by putting a sufficiently large number of these antenna elements in an array.

For beam forming synthesis, an adaptation of the classical least squares synthesis was proposed. This method yields a solution with an improved amplitude profile. Truncation of the SVD yields an approximate pseudoinverse resulting in excitation values with much better taper efficiency while the radiated pattern closely resembles the pattern obtained with the classical least squares synthesis.

\section{ACKNOWLEDGEMENT}

This work is part of the FlySmart project which is supported by the Dutch Ministry of Economic Affairs (SenterNovem project number IS053030). The FlySmart project is part of the Eureka EURIPIDES project SMART. 


\section{REFERENCES}

[1] P. Jorna, H. Schippers, J. Verpoorte, "Beam Synthesis for Conformal Array Antennas with Efficient Tapering”, Proceedings of 5th European Workshop on Conformal Antennas, Bristol, September 11-12, 2007.

[2] L. Zhuang, A. Meijerink, C. G. H. Roeloffzen, D. A. I. Marpaung, J. Peña Hevilla, W. van Etten, R. G. Heideman, A. Leinse, M.

Hoekman, "Phased array receive antenna steering system using a ring resonator-based optical beam forming network and filter-based optical SSB-SC modulation," Proceedings of the International Topical Meeting on Microwave Photonics (MWP'2007), Victoria, BC Canada, 3-5 October 2007, p. Th-3.3, pp. 88-91.

[3] The Radio Regulations, edition of 2004, contain the complete texts of the Radio Regulations as adopted by the World Radiocommunication Conference (Geneva, 1995) (WRC-95) and subsequently revised and adopted by the World Radiocommunication Conference (Geneva, 1997) (WRC-97), the World Radio-communication Conference (Istanbul, 2000) (WRC-2000), and the World Radio-communication Conference (Geneva, 2003) (WRC-03) including all Appendices, Resolutions,

Recommendations and ITU-R Recommendations incorporated by reference.

[4] RECOMMENDATION ITU-R M.1643, Technical and operational requirements for aircraft earth stations of aeronautical mobilesatellite service including those using fixed satellite service network transponders in the band $14-14.5 \mathrm{GHz}$ (Earth-to-space), 2003

[5] ETSI EN 302186 v1.1.1 (2004-01); Satellite Earth Stations and Systems (SES); Harmonised European Norms for satellite mobile Aircraft Earth Stations (AESs) operating in the 11/12/14 GHz frequency bands covering essential requirements under article 3.2 of the R\&TTE directive

[6] EUROCAE ED-14E; Environmental Conditions and Test procedures for Airborne Equipment, March 2005.

[7] F. Croq and D. M. Pozar, "Millimeter wave design of wide-band aperture-coupled stacked microstrip antennas," IEEE Trans. Antennas Propagat., vol. 39, pp. 1770-1776, Dec. 1991

[8] S. D. Targonski, R. B. Waterhouse, D. M. Pozar, "Design of wideband aperture-stacked patch microstrip antennas ", IEEE Transactions on Antennas and Propagation, vol. 46, no. 9, Sep. 1998, pp. 1245-1251.

[9] R. B. Waterhouse, "Design of probe-fed stacked patches", IEEE Transactions on Antennas and Propagation, vol. 47, no. 12, Dec. 1999, pp. 1780-1784.

[10] http://www.ansoft.com

[11] L. Josefsson and P. Persson, "Conformal Array Antenna Theory and Design”, IEEE Press, John Wiley \& Sons, Hoboken, New Jersey, 2006.

[12] L.I. Vaskelainen, "Iterative Least-Squares Synthesis Methods for Conformal Array Antennas with Optimized Polarization and Frequency Properties", IEEE Transactions on Antennas and Propagation, Vol. 45, No. 7, July 1997.

[13] L.I. Vaskelainen, "Phase Synthesis of Conformal Array Antennas", IEEE Transactions on Antennas and Propagation, Vol. 48, No. 6, June 2000.

[14] L.I. Vaskelainen, "Constrained Least-Squares Optimization in Conformal Array Antenna Synthesis", IEEE Transactions on Antennas and Propagation, Vol. 55, No. 3, March 2007.

[15] H. Lebret and S. Boyd, "Antenna Array Pattern Synthesis via Convex Optimization”, IEEE Transactions on Signal Processing, Vol. 45, No. 3, March 1997.

[16] S. Boyd and L. Vandenberghe, "Convex Optimization”, Cambridge University Press, 2004.

[17] P. Kassakian, "Magnitude Least-Squares Fitting via Semidefinite Programming with Applications to Beamforming and Multidimensional Filter Design", Proceedings IEEE International Conference on Acoustics, Speech and Signal Processing, Vol. 3, March 2005.

[18] F. Wang, V. Balakrishnan, P. Zhou, J. Chen, R. Yang C. Frank, "Optimal Array Pattern Synthesis Using Semidefinite
Programming”, IEEE Transactions on Signal Processing, Vol. 51, Issue. 5, May 2003.

[19] G.H.Golub and C.F. van Loan, "Matrix Computations", 3rd edition, The John Hopkins University Press, Baltimore, 1996.

[20] R.C. Hansen, "A One-Parameter Circular Aperture Distribution with Narrow Beamwidth and Low Sidelobes", IEEE Transactions on Antennas and Propagation, Vol. 24, Issue 4, July 1976.

[21] J.R. James, P.S. Hall, and C. Wood, "Microstrip Antenna Theory and Design", New York, Peter Peregrinus, 1981.

[22] P.G. Elliot, "Conformal Array Beam Synthesis and Taper Efficiency Comparisons", Proceedings Antennas, Radar, and Wave Propagation (ARP), 2005. 